\title{
S2 Figure
}
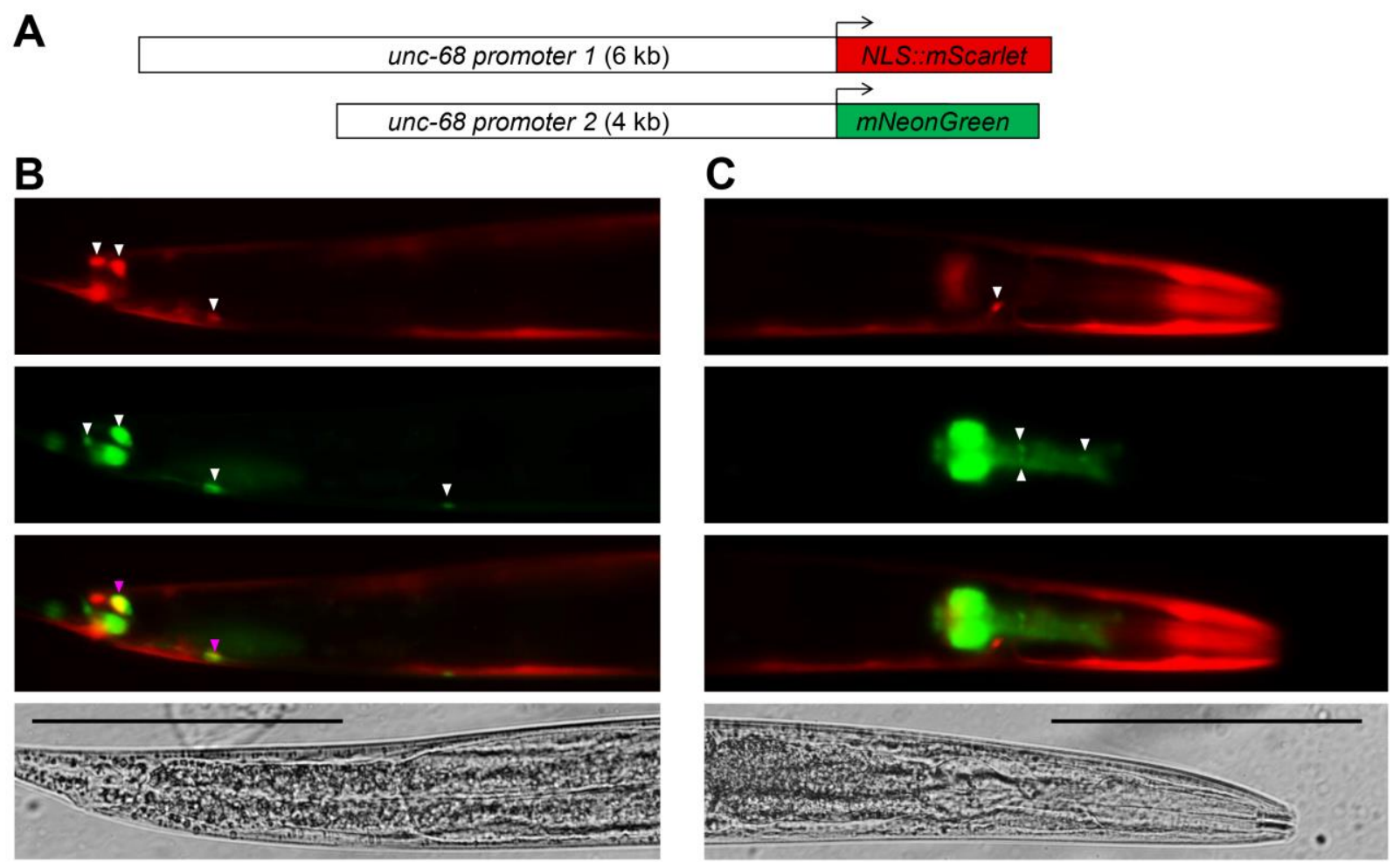

S2 Figure. unc-68 promoter analysis with two-color reporters

(A) Schematic of the two co-injected reporter genes.

$(B, C)$ Representative micrographs of tail $(B)$ and head $(C)$ regions. From top to bottom: red channel, green channel, merged red/green channel, and DIC. Similar results were obtained in three independent transgenic lines. The Nuclear Localization Signal (NLS) in the [unc-68p1::NLS:::mScarlet] did not generate a strong nuclear accumulation of the red fluorescent protein. White arrowheads: cells expressing either reporter. Pink arrowheads: cells expressing both reporters. Scale bars: $100 \mu \mathrm{m}$ 